\title{
TAIL ASYMPTOTIC BEHAVIOR OF THE SUPREMUM OF A CLASS OF CHI-SQUARE PROCESSES
}

\author{
LANPENG JI, PENG LIU, AND STEPHAN ROBERT
}

\begin{abstract}
We analyze in this paper the supremum of a class of chi-square processes over non-compact intervals, which can be seen as a multivariate counterpart of the generalized weighted Kolmogorov-Smirnov statistic. The boundedness and the exact tail asymptotic behavior of the supremum are derived. As examples, the chi-square process generated from the Brownian bridge and the fractional Brownian motion are discussed.
\end{abstract}

Key Words: chi-square process; exact asymptotics; Brownian bridge

AMS Classification: Primary 60G15; secondary 60G70

\section{INTRODUCTION}

Let $X(t), t \geq 0$, be a Gaussian process with almost surely (a.s.) continuous sample paths. For a sequence of constants $\left\{b_{i}\right\}_{i=1}^{n}$ satisfying

$$
1=b_{1}=\cdots=b_{k}>b_{k+1} \geq \cdots \geq b_{n}>0
$$

we define the chi-square process as

$$
\chi_{\boldsymbol{b}}^{2}(t)=\sum_{i=1}^{n} b_{i}^{2} X_{i}^{2}(t), \quad t \geq 0,
$$

where $X_{i}$ 's are independent copies of $X$. The supremum of chi-square process appears naturally as limiting test statistic in various statistical models; see, e.g., [1, 2]. It also plays an important role in reliability applications in the engineering sciences, see $[3,4]$ and the references therein.

Of interest in applied probability and statistics is the tail asymptotics of

$$
\mathbb{P}\left\{\sup _{t \in \mathcal{T}} \chi_{\boldsymbol{b}}^{2}(t)>u\right\}, \quad u \rightarrow \infty
$$

for an interval $\mathcal{T} \subset \mathbb{R}_{+}$, provided that

$$
\sup _{t \in \mathcal{T}} \chi_{\boldsymbol{b}}^{2}(t)<\infty \quad \text { a.s.. }
$$

Numerous contributions have been devoted to the study of the tail asymptotics of the supremum of chi-square processes over compact intervals $\mathcal{T}$; see, e.g., $[3,5]$ and the references therein, where the technique used is to transform the supremum of chi-square process into the supremum of a special Gaussian random field. We refer to, e.g., $[6,7,8,9]$ for more discussions on the tail asymptotics (or excursion probability) of Gaussian and related fields.

In this paper, we are interested in the analysis of the supremum of a class of weighted locally stationary chi-square processes defined by

$$
\sup _{t \in \mathcal{T}} \frac{\chi_{\boldsymbol{b}}^{2}(t)}{w^{2}(t)}, \quad \text { with } \mathcal{T}=(0,1) \text { or }(0,1]
$$

Date: July 1, 2019. 
where $w(\cdot)$ is some positive continuous function definable on the non-compact set $\mathcal{T}$, and the generic process $X$ is the locally stationary Gaussian process. More precisely, $X(t), t \in \mathcal{T}$, is a centered Gaussian process with a.s. continuous sample paths, unit variance and correlation function $r(\cdot, \cdot)$ satisfying

$$
\lim _{h \rightarrow 0} \frac{1-r(t, t+h)}{K^{2}(|h|)}=C(t)
$$

uniformly in $t \in I$, for all the compact interval $I$ in $\mathcal{T}$, where $K(\cdot)$ is a positive regularly varying function at 0 with index $\alpha / 2 \in(0,1]$, and $C(\cdot)$ is a positive continuous function satisfying

$$
\lim _{t \rightarrow 0} C(t)=\infty \text { or } \lim _{t \rightarrow 1} C(t)=\infty .
$$

We refer to [10] for more discussions on such locally stationary Gaussian processes.

Our motivation for considering the supremum of the weighted locally stationary chi-square processes over the noncompact interval $\mathcal{T}=(0,1)$ or $(0,1]$ is from its potential applications in statistics. For instance, in its univariate framework (with $n=1$ ) the following generalized weighted Kolmogorov-Smirnov statistic

$$
W_{w}:=\sup _{t \in(0,1)} \frac{|\bar{B}(t)|}{w(t)}, \quad \text { with } \bar{B}(t)=\frac{B(t)}{\sqrt{t(1-t)}}, \quad t \in(0,1),
$$

has been discussed in the statistics literature, see, e.g., [11], where $B$ is the standard Brownian bridge with variance function $\operatorname{Var}(B(t))=t(1-t), t \in[0,1]$ and $w$ is a suitably chosen weight function such that

$$
W_{w}<\infty \quad \text { a.s.. }
$$

We refer to $[11,12,13,14]$ for further discussions on the generalized weighted Kolmogorov-Smirnov statistic. An interesting theoritical question is to find sufficient and necessary conditions on $w$ so that the a.s. finiteness of (4) holds. It is shown in [11][Theorem 3.3, Theorem 4.2.3] (see also [12][Theorem 26.3]) that

$$
W_{w}<\infty \text { a.s. } \Leftrightarrow \int_{0}^{1} \frac{1}{t(1-t)} e^{-c w^{2}(t)} d t<\infty \text { for some } c>0 .
$$

One of the main results displayed in Theorem 3.1 shows necessary and sufficient conditions on the weight function $w$ under which it holds that

$$
\sup _{t \in \mathcal{T}} \frac{\chi_{\boldsymbol{b}}^{2}(t)}{w^{2}(t)}<\infty \quad \text { a.s.. }
$$

This extends the result of (5). Furthermore, for certain $w$ satisfying (6) we derive in Theorem 3.3 the exact asymptotics of

$$
\mathbb{P}\left\{\sup _{t \in \mathcal{T}} \frac{\chi_{\boldsymbol{b}}^{2}(t)}{w^{2}(t)}>u\right\}, \quad u \rightarrow \infty .
$$

As an important application of Theorem 3.3, we obtain in Corollary 3.4 the tail asymptotics of the supremum of the chi-square process generated from the Brownian bridge. It is worth mentioning that this tail asymptotic result is new even for the univariate (i.e., $n=1$ ) case. As a second example, the chi-square process generated by the fractional Brownian motion is discussed.

We expect that the derived results will have interesting applications in statistics or beyond.

Organization of the rest of the paper: In Section 2 we present a preliminary result which is a tailored version of Theorem A.1 in [10]. The main results are given in Section 3, followed by examples. All the proofs are displayed in Section 4. 


\section{Preliminaries}

This section concerns a result derived in [10], which is crucial for the derivation of (6). Based on the discussions therein, we shall consider $\int_{0}^{1 / 2}(C(s))^{1 / \alpha} d s=\infty$ or $\int_{1 / 2}^{1}(C(s))^{1 / \alpha} d s=\infty$. For this purpose, of crucial importance is the following function

$$
f(t)=\int_{1 / 2}^{t}(C(s))^{1 / \alpha} d s, \quad t \in(0,1)
$$

We denote by $\overleftarrow{f}(t), t \in(f(0), f(1))$ the inverse function of $f(t), t \in(0,1)$. Further, for any $d>0$, let $s_{j, d}^{(1)}=\overleftarrow{f}(j d)$ $j \in \mathbb{N} \cup\{0\}$ if $f(1)=\infty$, and let $s_{j, d}^{(0)}=\overleftarrow{f}(-j d), j \in \mathbb{N} \cup\{0\}$ if $f(0)=-\infty$. Denote $\Delta_{j, d}^{(1)}=\left[s_{j-1, d}^{(1)}, s_{j, d}^{(1)}\right], j \in \mathbb{N}$ and $\Delta_{j, d}^{(0)}=\left[s_{j, d}^{(0)}, s_{j-1, d}^{(0)}\right], j \in \mathbb{N}$, which give a partition of $[1 / 2,1)$ in the case $f(1)=\infty$ and a partition of $(0,1 / 2]$ in the case $f(0)=-\infty$, respectively. Moreover, let $q(u)=\overleftarrow{K}\left(u^{-1 / 2}\right)$ be the inverse function of $K(\cdot)$ at point $u^{-1 / 2}$ (assumed to exist asymptotically).

The following (scenario-dependent) restrictions on the positive continuous weight function $w^{2}$ and the correlation function $r(\cdot, \cdot)$ of $X$ play a crucial role. Let therefore $S \in\{0,1\}$.

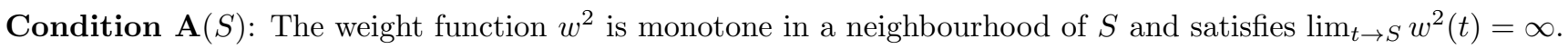

Condition $\mathbf{B}(S)$ : Suppose that there exists some constant $d_{0}>0$ such that

$$
\limsup _{j \rightarrow \infty} \sup _{t \neq s \in \Delta_{j, d_{0}}^{(S)}} \frac{1-r(t, s)}{K^{2}(|f(t)-f(s)|)}<\infty,
$$

and when $\alpha=2$ and $k=1$, assume further

$$
K^{2}(|t|)=O\left(t^{2}\right), \quad t \rightarrow 0
$$

Condition $\mathbf{C}(S)$ : Suppose that there exists some constant $d_{0}>0$ such that

$$
\liminf _{j \rightarrow \infty} \inf _{t \neq s \in \Delta_{j, d_{0}}^{(S)}} \frac{1-r(t, s)}{K^{2}(|f(t)-f(s)|)}>0 .
$$

Moreover, there exist $j_{0}, l_{0} \in \mathbb{N}, M_{0}, \beta>0$, such that for $j \geq j_{0}, l \geq l_{0}$,

$$
\sup _{s \in \Delta_{j+l, d_{0}}^{(S)}, t \in \Delta_{j, d_{0}}^{(S)}}|r(s, t)|<M_{0} l^{-\beta} .
$$

For the subsequent discussions we present a tailored version of Theorem A.1 of [10], focusing on $|f(S)|=\infty$. We define

$$
I_{w}(S)=\left|\int_{1 / 2}^{S}(C(t))^{1 / \alpha} \frac{(w(t))^{k-2}}{q\left(w^{2}(t)\right)} e^{-\frac{w^{2}(t)}{2}} d t\right|
$$

Theorem 2.1. Let $X(t), t \in(0,1)$, be a centered locally stationary Gaussian process with a.s. continuous sample paths, unit variance and correlation function $r(\cdot, \cdot)$ satisfying $(3)$ and $r(s, t)<1$ for $s \neq t \in(0,1)$. Suppose further that, for $S=0$ or 1 , we have $|f(S)|=\infty$ and $\mathbf{A}(S), \mathbf{B}(S), \mathbf{C}(S)$ are satisfied. Then

$$
\mathbb{P}\left\{\chi_{\boldsymbol{b}}^{2}(t) \leq w^{2}(t) \quad \text { ultimately as } t \rightarrow S\right\}=0, \quad \text { or } \quad 1
$$

according to

$$
I_{w}(S)=\infty, \quad \text { or } \quad<\infty .
$$




\section{MAin Results}

In this section, we first give a criteria for (6) to hold and then display the exact asymptotics of (7) for different types of $w$ such that (6) is valid.

3.1. Analysis of (6). Denote by $E(0)=(0,1 / 2]$ and $E(1)=[1 / 2,1)$. Recall that $S \in\{0,1\}$. Under the conditions of Theorem 2.1, we have that if $I_{w}(S)<\infty$, then

$$
\sup _{t \in E(S)} \frac{\chi_{\boldsymbol{b}}^{2}(t)}{w^{2}(t)}<\infty \quad \text { a.s. }
$$

however, when $I_{w}(S)=\infty$ we only see that

$$
\sup _{t \in E(S)} \frac{\chi_{\boldsymbol{b}}^{2}(t)}{w^{2}(t)} \geq 1 \quad \text { a.s.. }
$$

Apparently, the above is not informative for the claim in (6). On the other hand, it is easily shown that

$$
\sup _{t \in E(S)} \frac{\chi_{\boldsymbol{b}}^{2}(t)}{w^{2}(t)}<\infty \text { a.s. } \Leftrightarrow \sup _{t \in E(S)} \frac{|X(t)|}{w(t)}<\infty \text { a.s. }
$$

which means that, instead of the condition $I_{w}(S)=\infty$ in Theorem 2.1, a more accurate condition that is independent of $n, k$ should be possible to ensure that (6) holds. Inspired by this fact, we provide below a sufficient and necessary condition for (6) to hold.

Define, for any constant $c>0$ and any positive continuous function $w$

$$
J_{c, w}(S)=\left|\int_{1 / 2}^{S}(C(t))^{1 / \alpha} e^{-c w^{2}(t)} d t\right| .
$$

Below is our first principal result, a criterion for (6), which is a generalization of (5).

Theorem 3.1. Under the conditions of Theorem 2.1 we have

$$
\sup _{t \in E(S)} \frac{\chi_{\boldsymbol{b}}^{2}(t)}{w^{2}(t)}<\infty \text { a.s. } \Leftrightarrow J_{c, w}(S)<\infty \text { for some } c>0 .
$$

Next we illustrate the criteria presented in Theorem 3.1 by an example of a weighted chi-square process with generic process being the normalized standard Brownian bridge, which further provides us with a clear comparison between $I_{w}(S)$ and $J_{c, w}(S)$.

Example 3.2. Let $X(t)=\bar{B}(t), t \in(0,1)$, and, with $\rho_{1}>0, \rho_{2} \in \mathbb{R}$, define

$$
w_{\rho_{1}, \rho_{2}}^{2}(t)=2 \rho_{1} \ln \ln \left(\frac{e^{2}}{t(1-t)}\right)+2 \rho_{2} \ln \ln \ln \left(\frac{e^{2}}{t(1-t)}\right), t \in(0,1) .
$$

First note that for the normalized standard Brownian bridge

$$
\lim _{h \rightarrow 0} \frac{1-\mathbb{E}(\bar{B}(t) \bar{B}(t+h))}{|h|}=\frac{1}{2 t(1-t)}
$$

holds uniformly in $t \in I$, for any compact interval $I$ in $(0,1)$. This means that $\bar{B}$ is a locally stationary Gaussian process with

$$
K(h)=\sqrt{|h|}, \quad \alpha=1, \quad q(u)=u^{-1} .
$$

Furthermore,

$$
f(t)=\int_{1 / 2}^{t} \frac{1}{2 s(1-s)} d s=\frac{1}{2} \ln \left(\frac{t}{1-t}\right)
$$


implying that $f(1)=-f(0)=\infty$. Moreover, by the proof of Corollary 2.6 in [10] we have that conditions $\mathbf{B}(\mathbf{S})$ and $\mathbf{C}(\mathbf{S})$ are satisfied by $\bar{B}(t), t \in(0,1)$, and $\mathbb{E}(\bar{B}(t), \bar{B}(s))<1$ for $s \neq t, s, t \in(0,1)$. Thus, all the conditions of Theorem 3.1 are fulfilled.

Next, on one hand, we have

$$
\frac{1}{t(1-t)}\left(w_{\rho_{1}, \rho_{2}}(t)\right)^{k} e^{-\frac{w_{\rho_{1}, \rho_{2}}^{2}(t)}{2}} \sim \frac{Q}{t(1-t)\left(\ln \left(\frac{1}{t(1-t)}\right)\right)^{\rho_{1}}\left(\ln \ln \left(\frac{e^{2}}{t(1-t)}\right)\right)^{\rho_{2}-k / 2}}
$$

as $t \rightarrow 0$ or $t \rightarrow 1$, with $Q$ some positive constant. Thus, elementary calculations show that

$$
I_{w}(0)=I_{w}(1)=\int_{1 / 2}^{1} \frac{\left(w_{\rho_{1}, \rho_{2}}(t)\right)^{k}}{t(1-t)} e^{-\frac{w_{\rho_{1}, \rho_{2}}^{2}(t)}{2}} d t<\infty
$$

holds if and only if

$$
\rho_{1}>1, \quad \text { or } \rho_{1}=1 \text { and } \rho_{2}>1+k / 2 \text {. }
$$

On the other hand, we can show that the functions $w_{\rho_{1}, \rho_{2}}(t)$ satisfying that $\exists c>0$ such that $J_{c, w}(S)<\infty$ are not restricted to the ones satisfying (12). In fact, since for any $\rho_{1}>0$ there exists some $c$ such that $\rho_{1}>\frac{1}{2 c}$, we have that

$$
\begin{aligned}
J_{c, w}(0)=J_{c, w}(1) & =\int_{1 / 2}^{1} \frac{1}{t(1-t)} e^{-c w_{\rho_{1}, \rho_{2}}^{2}(t)} d t \\
& \leq \int_{1 / 2}^{1} \frac{1}{t(1-t)\left(\ln \left(\frac{1}{t(1-t)}\right)\right)^{2 c \rho_{1}}\left(\ln \ln \left(\frac{e^{2}}{t(1-t)}\right)\right)^{2 c \rho_{2}}} d t<\infty
\end{aligned}
$$

holds for any $\rho_{2} \in \mathbb{R}$. Thus, we conclude from Theorem 3.1 that

$$
\sup _{t \in(0,1)} \frac{\chi_{b}^{2}(t)}{w_{\rho_{1}, \rho_{2}}^{2}(t)}<\infty \text { a.s. }
$$

holds for any $\rho_{1}>0$ and $\rho_{2} \in \mathbb{R}$.

3.2. Asymptotics of (7). For those $w$ such that (6) holds, of interest is the exact tail asymptotic behavior of $\sup _{t \in \mathcal{T}} \frac{\chi_{b}^{2}(t)}{w^{2}(t)}$. Actually, as we have seen, the behavior of $w$ around 0 and 1 plays a crucial role for the finiteness in (6). However, this does not apply to the tail asymptotics of $\sup _{t \in \mathcal{T}} \frac{\chi_{b}^{2}(t)}{w^{2}(t)}$. It turns out that only the probability mass in the neighborhood of minimizer of $w$ contribute to the tail asymptotics. As discussed in [15], the weight function is introduced when constructing the Goodness-of-Fit test which is intended to emphasize a specific region of the domain. With these motivations, for the tail asymptotics we shall consider the following two types of $w$ :

Assumption F1: The function $w$ attains its minimum at finite distinct inner points $\left\{t_{i}\right\}_{i=1}^{m}$ of $\mathcal{T}$, and

$$
w\left(t_{i}+t\right)=w\left(t_{i}\right)+a_{i}\left|t_{i}\right|^{\beta_{i}}(1+o(1)), \quad t \rightarrow t_{i}
$$

holds for some positive constants $a_{i}, \beta_{i}>0, i=1,2, \ldots, m$.

Assumption F2: The function $w$ attains its minimum at all points on disjoint intervals $\left[c_{i}, d_{i}\right] \subseteq \mathcal{T}, i=1,2, \ldots, m$ (i.e., $w$ is a constant on these intervals).

Under assumption $\mathbf{F 1}$, we need additional conditions which are stated below. Recall $q(u)=\overleftarrow{K}\left(u^{-1 / 2}\right)$. It follows that $q(u)$ is a regularly varying function at infinity with index $-1 / \alpha$ which can be further expressed as $q(u)=$ $u^{-1 / \alpha} L\left(u^{-1 / 2}\right)$, with $L(\cdot)$ a slowly varying function at 0 . Denote further $\beta=\max _{1 \leq i \leq m} \beta_{i}$. According to the values of $L\left(u^{-1 / 2}\right)$ as $u \rightarrow \infty$, we consider the following three scenarios:

C1 $(\beta): \beta>\alpha$, or $\beta=\alpha$ and $\lim _{u \rightarrow \infty} L\left(u^{-1 / 2}\right)=0$; 
$\mathbf{C 2}(\beta): \beta=\alpha$ and $\lim _{u \rightarrow \infty} L\left(u^{-1 / 2}\right)=\mathcal{L} \in(0, \infty)$

C3( $\beta): \beta<\alpha$, or $\beta=\alpha$ and $\lim _{u \rightarrow \infty} L\left(u^{-1 / 2}\right)=\infty$.

Before displaying our results, we introduce two important constants. One is the Pickands constant defined by

$$
\mathcal{H}_{2 H}=\lim _{S \rightarrow \infty} \frac{1}{S} \mathbb{E}\left(\exp \left(\sup _{t \in[0, S]}\left(\sqrt{2} B_{H}(t)-t^{2 H}\right)\right)\right),
$$

with $B_{H}(t), t \in \mathbb{R}$, a standard fractional Brownian motion ( $\mathrm{fBm}$ ) defined on $\mathbb{R}$ with Hurst index $H \in(0,1]$. And the other one is the Piterbarg constant defined by

$$
\mathcal{P}_{2 H}^{d}=\lim _{\lambda \rightarrow \infty} \mathbb{E}\left(\exp \left(\sup _{t \in[-\lambda, \lambda]}\left(\sqrt{2} B_{H}(t)-(1+d)|t|^{2 H}\right)\right)\right), \quad d>0 .
$$

We refer to [16] for the properties and generalizations of the Pickands-Piterbarg type constants. In what follows, $\alpha$ will play a similar role as $2 H$. Moreover, We shall use the standard notation for asymptotic equivalence of two functions $f$ and $h$. Specifically, we write $f(x) \sim h(x)$, if $\lim _{x \rightarrow a} f(x) / h(x)=1(a \in \mathbb{R} \cup\{\infty\})$, and further, write $f(x)=o(h(x))$, if $\lim _{x \rightarrow a} f(x) / h(x)=0$.

Let $K=\left\{1 \leq i \leq m: \beta_{i}=\beta\right\}$ and $K^{c}=\left\{1 \leq i \leq m: \beta_{i}<\beta\right\}$. Below is our second principal result.

Theorem 3.3. Let $\frac{\chi_{b}^{2}(t)}{w^{2}(t)}, t \in \mathcal{T}$, be the weighted locally stationary chi-square process considered in Theorem 2.1 such that (6) holds. We have:

(i). If $\mathbf{F} 1$ is satisfied, then, as $u \rightarrow \infty$,

$$
\mathbb{P}\left\{\sup _{t \in \mathcal{T}} \frac{\chi_{\boldsymbol{b}}^{2}(t)}{w^{2}(t)}>u\right\} \sim\left(\prod_{i=k+1}^{n}\left(1-b_{i}^{2}\right)^{-1 / 2}\right) \mathcal{M}(u) \Upsilon_{k}\left(w^{2}\left(t_{1}\right) u\right),
$$

where (with the convention $\prod_{i=p}^{q}=1$ if $q<p$ )

$$
\Upsilon_{k}(u):=\mathbb{P}\left\{\chi_{k, \mathbf{1}}^{2}(0)>u\right\}=\frac{2^{(2-k) / 2}}{\Gamma(k / 2)} u^{k / 2-1} \exp \left(-\frac{u}{2}\right), \quad u>0,
$$

and

$$
\mathcal{M}(u)= \begin{cases}2\left(\sum_{i \in K} a_{i}^{-1 / \beta}\left(C\left(t_{i}\right)\right)^{1 / \alpha}\right)\left(w\left(t_{1}\right)\right)^{2 / \alpha-1 / \beta} \Gamma(1 / \beta+1) \mathcal{H}_{\alpha}(q(u))^{-1} u^{-1 / \beta}, & \text { for } \boldsymbol{C 1}(\beta), \\ \sum_{i \in K} \mathcal{P}_{\alpha}^{a_{i}\left(w\left(t_{1}\right) C\left(t_{i}\right)\right)^{-1} \mathcal{L}^{\alpha}}+\sharp K^{c}, & \text { for } \boldsymbol{C 2}(\beta), \\ m, & \text { for } \boldsymbol{C 3}(\beta) .\end{cases}
$$

(ii). If $\mathbf{F 2}$ is satisfied, then, as $u \rightarrow \infty$,

$$
\mathbb{P}\left\{\sup _{t \in \mathcal{T}} \frac{\chi_{\boldsymbol{b}}^{2}(t)}{w^{2}(t)}>u\right\} \sim\left(\prod_{i=k+1}^{n}\left(1-b_{i}^{2}\right)^{-1 / 2}\right)\left(\sum_{j=1}^{m} \int_{c_{j}}^{d_{j}}(C(t))^{1 / \alpha} d t\right) \mathcal{H}_{\alpha}\left(q\left(w^{2}\left(c_{1}\right) u\right)\right)^{-1} \Upsilon_{k}\left(w^{2}\left(c_{1}\right) u\right) .
$$

We conclude this section with two applications of Theorem 3.3. Full proofs of Corollaries 3.4 and 3.5 can be found in [17].

Corollary 3.4. Let $\frac{\chi_{b}^{2}(t)}{w_{\rho_{1}, \rho_{2}}^{2}(t)}, t \in(0,1)$, with $\rho_{1}>0$ and $\rho_{2} \in \mathbb{R}$, be the weighted locally stationary chi-square process discussed in Example 3.2. We have, as $u \rightarrow \infty$, if $\rho_{2} \geq-\rho_{1} \ln \ln \left(4 e^{2}\right)$, then

$$
\mathbb{P}\left\{\sup _{t \in(0,1)} \frac{\chi_{\boldsymbol{b}}^{2}(t)}{w_{\rho_{1}, \rho_{2}}^{2}(t)}>u\right\} \sim\left(\prod_{i=k+1}^{n}\left(1-b_{i}^{2}\right)^{-1 / 2}\right) \mathcal{M}(u) \Upsilon_{k}\left(2 A_{1} u\right),
$$


where $A_{1}=\rho_{1} \ln \ln \left(4 e^{2}\right)+\rho_{2} \ln \ln \ln \left(4 e^{2}\right)$ and

$$
\mathcal{M}(u)= \begin{cases}2 A_{1} \sqrt{\frac{\pi \ln \left(4 e^{2}\right) \ln \ln \left(4 e^{2}\right)}{\rho_{1} \ln \ln \left(4 e^{2}\right)+\rho_{2}}} u^{1 / 2}, & \text { for } \rho_{2}>-\rho_{1} \ln \ln \left(4 e^{2}\right), \\ 2 \Gamma(1 / 4) A_{1}\left(\frac{\ln \ln \left(4 e^{2}\right)\left(\ln \left(4 e^{2}\right)\right)^{2}}{8 \rho_{1}}\right)^{1 / 4} u^{3 / 4}, & \text { for } \rho_{2}=-\rho_{1} \ln \ln \left(4 e^{2}\right),\end{cases}
$$

and if $\rho_{2}<-\rho_{1} \ln \ln \left(4 e^{2}\right)$, then

$$
\mathbb{P}\left\{\sup _{t \in(0,1)} \frac{\chi_{\boldsymbol{b}}^{2}(t)}{w_{\rho_{1}, \rho_{2}}^{2}(t)}>u\right\} \sim 2 A_{2}\left(\prod_{i=k+1}^{n}\left(1-b_{i}^{2}\right)^{-1 / 2}\right) \rho_{1}^{-1} Q \sqrt{-2 \pi \rho_{2}} u^{1 / 2} \Upsilon_{k}\left(2 A_{2} u\right),
$$

where $A_{2}=\rho_{2}\left(\ln \left(-\rho_{2}\right)-\ln \left(\rho_{1}\right)-1\right)$ and

$$
Q=\frac{1}{2 t_{1}-1} \ln \left(\frac{e^{2}}{t_{1}\left(1-t_{1}\right)}\right), \quad t_{1}=1 / 2+\sqrt{1 / 4-e^{2-e^{-\rho_{2} / \rho_{1}}}} .
$$

Next, we consider $B_{H}(t), t \geq 0$, to be the standard fBm with Hurst index $H \in(0,1)$ and covariance function

$$
\operatorname{Cov}\left(B_{H}(s), B_{H}(t)\right)=\frac{1}{2}\left(|s|^{2 H}+|t|^{2 H}-|s-t|^{2 H}\right), \quad s, t \geq 0 .
$$

Denote by $\bar{B}_{H}(t)=B_{H}(t) / t^{H}, t \in(0,1]$ the normalized standard fBm defined on $(0,1]$. Further, for any $\rho>0$ and $\varepsilon \in(0,1)$, we define

$$
w_{\rho, \varepsilon}^{2}(t)= \begin{cases}\rho \ln \ln \left(e^{2} / t\right), & \text { for } t \in(0, \varepsilon), \\ \rho \ln \ln \left(e^{2} / \varepsilon\right), & \text { for } t \in[\varepsilon, 1]\end{cases}
$$

We have the following result.

Corollary 3.5. Let $\frac{\chi_{b}^{2}(t)}{w_{\rho, \varepsilon}^{2}(t)}, t \in(0,1]$, be a weighted chi-square process with generic process $\bar{B}_{H}(t), t \in(0,1]$ and $w_{\rho, \varepsilon}^{2}$ given in (15). Then, we have, as $u \rightarrow \infty$,

$$
\begin{aligned}
\mathbb{P}\left\{\sup _{t \in(0,1)} \frac{\chi_{\boldsymbol{b}}^{2}(t)}{w_{\rho, \varepsilon}^{2}(t)}>u\right\} \sim & \left(\prod_{i=k+1}^{n}\left(1-b_{i}^{2}\right)^{-1 / 2}\right)(-\ln (\varepsilon))\left(\ln \ln \left(e^{2} / \varepsilon\right) \rho / 2\right)^{\frac{1}{2 H}} \\
& \times \mathcal{H}_{2 H} u^{\frac{1}{2 H}} \Upsilon_{k}\left(\rho \ln \ln \left(e^{2} / \varepsilon\right) u\right) .
\end{aligned}
$$

\section{Proofs}

This section is devoted to the proof of all the results presented in Section 3.

Proof of Theorem 3.1: Note that $t^{k / 2-1}(q(t))^{-1}$ is a positive regularly varying function at $\infty$ with index $\kappa=$ $k / 2-1+1 / \alpha \geq 0$. Thus, by Potter bound (e.g., [18])

$$
c_{1} t^{\kappa-1} \leq t^{k / 2-1}(q(t))^{-1} \leq c_{2} t^{\kappa+1}, \quad t \geq c_{3},
$$

holds for some constants $c_{1}, c_{2}, c_{3}>0$, which, together with the fact that $w^{2}(t) \rightarrow \infty$ as $t \rightarrow S$, leads to

$$
Q_{1} e^{-w^{2}(t)} \leq \frac{(w(t))^{k-2}}{q\left(w^{2}(t)\right)} e^{-\frac{w^{2}(t)}{2}} \leq Q_{2} e^{-\frac{w^{2}(t)}{3}}
$$

for all $t$ approaching $S$, with some positive constants $Q_{1}, Q_{2}$. Therefore, if $J_{c, w}(S)<\infty$ holds for some $c>0$, then, by $(16)$,

$$
I_{\sqrt{3 c} w}(S)<\infty .
$$

This together with ii) of Theorem 2.1 yields that

$$
\limsup _{t \rightarrow S} \frac{\chi_{\boldsymbol{b}}^{2}(t)}{w^{2}(t)} \leq 3 c \text { a.s. }
$$


showing that

$$
\sup _{t \in E(S)} \frac{\chi_{\boldsymbol{b}}^{2}(t)}{w^{2}(t)}<\infty \text { a.s. }
$$

On the other hand, if $J_{c, w}(S)=\infty$ for all $c>0$, then, by $(16), I_{\sqrt{c} w}(S)=\infty$. Thus, by iii) of Theorem 2.1

$$
\limsup _{t \rightarrow S} \frac{\chi_{\boldsymbol{b}}^{2}(t)}{w^{2}(t)} \geq c \text { a.s. }
$$

holds for all $c>0$, implying that

$$
\sup _{t \in E(S)} \frac{\chi_{\boldsymbol{b}}^{2}(t)}{w^{2}(t)}=\infty \text { a.s. }
$$

This completes the proof.

We present next two lemmas which will play key roles in the proof of Theorem 3.3; see [17] for proofs. Denote below $\mathcal{S} \subseteq \mathbb{R}$ to be any fixed interval.

Lemma 4.1. Let $\chi_{\boldsymbol{b}}^{2}(t), t \in \mathcal{S}$, be a chi-square process with generic centered Gaussian process $X$ which has a.s. continuous sample paths and variance function denoted by $\sigma_{X}^{2}(t)$. If

$$
\sup _{t \in \mathcal{S}} X(t)<\infty \quad \text { a.s. }
$$

then there exists some positive constant $Q$ such that for all $u>Q^{2}$ we have

$$
\mathbb{P}\left\{\sup _{t \in \mathcal{S}} \chi_{\boldsymbol{b}}^{2}(t)>u\right\} \leq \exp \left(-\frac{(\sqrt{u}-Q)^{2}}{2 \sup _{t \in \mathcal{S}} \sigma_{X}^{2}(t)}\right) .
$$

Lemma 4.2. Let $\chi_{\boldsymbol{b}}^{2}(t), t \in \mathcal{S}$, be a chi-square process with the generic centered locally stationary Gaussian process $X$ which has a.s. continuous sample paths. If further the correlation function of $X$ satisfies

$$
r(s, t)<1 \text { for any } s \neq t \in \mathcal{S},
$$

then, for any compact intervals $\mathcal{S}_{1}, \mathcal{S}_{2} \subset \mathcal{S}$ such that $\mathcal{S}_{1} \cap \mathcal{S}_{2}=\emptyset$ we have

$$
\mathbb{P}\left\{\sup _{t \in \mathcal{S}_{1}} \chi_{\boldsymbol{b}}^{2}(t)>u, \sup _{t \in \mathcal{S}_{2}} \chi_{\boldsymbol{b}}^{2}(t)>u\right\} \leq \exp \left(-\frac{(2 \sqrt{u}-Q)^{2}}{2(2+2 \eta)}\right) .
$$

for all $u>Q^{2}$, with some constant $Q>0$ and $\eta \in(0,1)$.

Proof of Theorem 3.3: Without loss of generality, we show the proof only for the case where $\mathcal{T}=(0,1)$.

(i). Let $\rho>0$ be a sufficiently small constant such that

$$
\left[t_{i}-\rho, t_{i}+\rho\right] \cap\left[t_{j}-\rho, t_{j}+\rho\right]=\emptyset, \quad \text { for all } i \neq j .
$$

Further, denote $\mathcal{T}_{\rho}=\mathcal{T} \backslash \bigcup_{i=1}^{m}\left[t_{i}-\rho, t_{i}+\rho\right]$. It follows from the Bonferroni inequality (e.g., [19]) that

$$
\begin{aligned}
& \sum_{i=1}^{m} p_{i}(u)+\mathbb{P}\left\{\sup _{t \in \mathcal{T}_{\rho}} \frac{\chi_{\boldsymbol{b}}^{2}(t)}{w^{2}(t)}>u\right\} \\
& \geq \mathbb{P}\left\{\sup _{t \in \mathcal{T}} \frac{\chi_{\boldsymbol{b}}^{2}(t)}{w^{2}(t)}>u\right\} \\
& \geq \sum_{i=1}^{m} p_{i}(u)-\sum_{1 \leq i<j \leq m} \mathbb{P}\left\{\sup _{t \in\left[t_{i}-\rho, t_{i}+\rho\right]} \frac{\chi_{\boldsymbol{b}}^{2}(t)}{w^{2}(t)}>u, \sup _{t \in\left[t_{j}-\rho, t_{j}+\rho\right]} \frac{\chi_{\boldsymbol{b}}^{2}(t)}{w^{2}(t)}>u\right\},
\end{aligned}
$$

where

$$
p_{i}(u)=\mathbb{P}\left\{\sup _{t \in\left[t_{i}-\rho, t_{i}+\rho\right]} \frac{\chi_{\boldsymbol{b}}^{2}(t)}{w^{2}(t)}>u\right\} .
$$


We first focus on the asymptotics of $p_{i}(u)$ as $u \rightarrow \infty$. Denote

$$
Y(t)=\frac{w\left(t_{1}\right)}{w(t)} X(t), \quad t \in \mathcal{T}
$$

We have

$$
p_{i}(u)=\mathbb{P}\left\{\sup _{t \in\left[t_{i}-\rho, t_{i}+\rho\right]} \sum_{l=1}^{n} b_{l}^{2} Y_{l}^{2}(t)>w^{2}\left(t_{1}\right) u\right\}, \quad 1 \leq i \leq m,
$$

where $\left\{Y_{l}\right\}_{i=1}^{n}$ is a sequence of independent copies of Gaussian process $Y$. It can be shown that, by $\mathbf{F} \mathbf{1}$, for any $i=1,2, \cdots, m$,

$$
\sigma_{Y}(t)=\sqrt{\mathbb{E}\left((Y(t))^{2}\right)}=\frac{w\left(t_{1}\right)}{w(t)}, \quad t \in\left[t_{i}-\rho, t_{i}+\rho\right],
$$

attains its maximum which is equal to 1 at the unique point $t_{i}$, and further

$$
\sigma_{Y}\left(t_{i}+t\right)=1-\frac{a_{i}}{w\left(t_{1}\right)}|t|^{\beta_{i}}(1+o(1)), \quad t \rightarrow 0 .
$$

Moreover, by (3)

$$
1-\operatorname{Corr}\left(Y\left(t_{i}+t\right), Y\left(t_{i}+s\right)\right)=C\left(t_{i}\right) K^{2}(|t-s|)(1+o(1)), \quad t \rightarrow 0
$$

Consequently, it follows from [4][Theorem 5.2] that, as $u \rightarrow \infty$,

$$
p_{i}(u) \sim\left(\prod_{l=k+1}^{n}\left(1-b_{l}^{2}\right)^{-1 / 2}\right) \mathcal{M}_{i}\left(\beta_{i}, u\right) \Upsilon_{k}\left(w^{2}\left(t_{1}\right) u\right),
$$

where $\Upsilon_{k}(\cdot)$ is given in (14) and

$$
\mathcal{M}_{i}\left(\beta_{i}, u\right)= \begin{cases}2 a_{i}^{-1 / \beta_{i}}\left(w\left(t_{1}\right)\right)^{2 / \alpha-1 / \beta_{i}}\left(C\left(t_{i}\right)\right)^{1 / \alpha} \Gamma\left(1 / \beta_{i}+1\right) \mathcal{H}_{\alpha}(q(u))^{-1} u^{-1 / \beta_{i}}, & \text { for } \mathbf{C 1}\left(\beta_{i}\right) \\ \mathcal{P}_{\alpha}^{a_{i}\left(w\left(t_{1}\right) C\left(t_{i}\right)\right)^{-1} \mathcal{L}^{\alpha}} & \text { for } \mathbf{C 2}\left(\beta_{i}\right) \\ 1, & \text { for } \mathbf{C 3}\left(\beta_{i}\right)\end{cases}
$$

In the sequel, we discuss the three scenarios $\mathbf{C 1}(\beta), \mathbf{C 2}(\beta), \mathbf{C 3}(\beta)$ one-by one.

$\underline{\mathbf{C 1}(\beta)}$. Using the fact that $\beta=\max _{i=1}^{m} \beta_{i}$, we have that

$$
\mathcal{M}_{j}\left(\beta_{j}, u\right)=o\left(\mathcal{M}_{i}\left(\beta_{i}, u\right)\right), \quad u \rightarrow \infty
$$

for any $i \in K$ and $j \in K^{c}$. This implies that

$$
\sum_{i=1}^{m} p_{i}(u) \sim \sum_{i \in K} p_{i}(u) \sim\left(\prod_{l=k+1}^{n}\left(1-b_{l}^{2}\right)^{-1 / 2}\right) \mathcal{M}(u) \Upsilon_{k}\left(w^{2}\left(t_{1}\right) u\right)
$$

where $\mathcal{M}(\cdot)$ is given in (15). On the other hand, it follows directly from Lemma 4.1 that

$$
\mathbb{P}\left\{\sup _{t \in \mathcal{T}_{\rho}} \frac{\chi_{\boldsymbol{b}}^{2}(t)}{w^{2}(t)}>u\right\} \leq \exp \left(-\frac{\inf _{t \in \mathcal{T}_{\rho}} w^{2}(t)(\sqrt{u}-Q)^{2}}{2}\right)
$$

holds for all $u>Q^{2}$, with $Q$ some positive constant. Since further, by $\mathbf{F 1}$,

$$
\inf _{t \in \mathcal{T}_{\rho}} w^{2}(t)>w^{2}\left(t_{1}\right)
$$

we have that

$$
\mathbb{P}\left\{\sup _{t \in \mathcal{T}_{\rho}} \frac{\chi_{\boldsymbol{b}}^{2}(t)}{w^{2}(t)}>u\right\}=o\left(\mathcal{M}(u) \Upsilon_{k}\left(w^{2}\left(t_{1}\right) u\right)\right), \quad u \rightarrow \infty
$$


Moreover, since for any $i \neq j$

$$
\begin{aligned}
& \mathbb{P}\left\{\sup _{t \in\left[t_{i}-\rho, t_{i}+\rho\right]} \frac{\chi_{\boldsymbol{b}}^{2}(t)}{w^{2}(t)}>u, \sup _{t \in\left[t_{j}-\rho, t_{j}+\rho\right]} \frac{\chi_{\boldsymbol{b}}^{2}(t)}{w^{2}(t)}>u\right\} \\
& \quad \leq \mathbb{P}\left\{\sup _{t \in\left[t_{i}-\rho, t_{i}+\rho\right]} \chi_{\boldsymbol{b}}^{2}(t)>w^{2}\left(t_{1}\right) u, \sup _{t \in\left[t_{j}-\rho, t_{j}+\rho\right]} \chi_{\boldsymbol{b}}^{2}(t)>w^{2}\left(t_{1}\right) u\right\} .
\end{aligned}
$$

we have from Lemma 4.2 that, for all $u$ large,

$$
\mathbb{P}\left\{\sup _{t \in\left[t_{i}-\rho, t_{i}+\rho\right]} \frac{\chi_{\boldsymbol{b}}^{2}(t)}{w^{2}(t)}>u, \sup _{t \in\left[t_{j}-\rho, t_{j}+\rho\right]} \frac{\chi_{\boldsymbol{b}}^{2}(t)}{w^{2}(t)}>u\right\} \leq \exp \left(-\frac{\left(2 w\left(t_{1}\right) \sqrt{u}-Q_{i, j}\right)^{2}}{2(2+2 \eta)}\right), \quad 1 \leq i<j \leq m,
$$

with $Q_{i, j}$ 's some positive constants and $\eta \in(0,1)$. Therefore, as $u \rightarrow \infty$,

$$
\sum_{1 \leq i<j \leq m} \mathbb{P}\left\{\sup _{t \in\left[t_{i}-\rho, t_{i}+\rho\right]} \frac{\chi_{\boldsymbol{b}}^{2}(t)}{w^{2}(t)}>u, \sup _{t \in\left[t_{j}-\rho, t_{j}+\rho\right]} \frac{\chi_{\boldsymbol{b}}^{2}(t)}{w^{2}(t)}>u\right\}=o\left(\mathcal{M}(u) \Upsilon_{k}\left(w^{2}\left(t_{1}\right) u\right)\right) .
$$

Combining (21)-(23) with (19) we establish the claim of $\mathbf{C 1}(\beta)$.

C2 $(\beta)$. In this case, we have that (20) holds with

$$
\mathcal{M}_{i}\left(\beta_{i}, u\right)=\left\{\begin{array}{cc}
\mathcal{P}_{\alpha}^{a_{i}\left(w\left(t_{1}\right) C\left(t_{i}\right)\right)^{-1} \mathcal{L}^{\alpha},} & i \in K \\
1, & i \in K^{c} .
\end{array}\right.
$$

Consequently,

$$
\sum_{i=1}^{m} p_{i}(u) \sim\left(\prod_{l=k+1}^{n}\left(1-b_{l}^{2}\right)^{-1 / 2}\right)\left(\sum_{i \in K} \mathcal{P}_{\alpha}^{a_{i}\left(w\left(t_{1}\right) C\left(t_{i}\right)\right)^{-1} \mathcal{L}^{\alpha}}+\sharp K^{c}\right) \Upsilon_{k}\left(w^{2}\left(t_{1}\right) u\right) .
$$

Note that (22) and (23) still hold. Similarly as the case $\mathbf{C 1}(\beta)$, we establish the claim of $\mathbf{C 2}(\beta)$.

C3 $(\beta)$. In this case, we have that (20) holds with

$$
\mathcal{M}_{i}\left(\beta_{i}, u\right)=1, \quad 1 \leq i \leq m
$$

Consequently,

$$
\sum_{i=1}^{m} p_{i}(u) \sim m\left(\prod_{l=k+1}^{n}\left(1-b_{l}^{2}\right)^{-1 / 2}\right) \Upsilon_{k}\left(w^{2}\left(t_{1}\right) u\right) .
$$

Similarly as before, the claim of $\mathbf{C 3}(\beta)$ follows.

(ii). By F2 we have for any sufficiently small $\varepsilon>0$ it holds that

$$
\inf _{t \in \mathcal{T}_{\varepsilon}} w(t)>w\left(c_{1}\right), \quad \text { with } \mathcal{T}_{\varepsilon}=\mathcal{T} \backslash \bigcup_{i=1}^{m}\left[c_{i}-\varepsilon, d_{i}+\varepsilon\right]
$$

Similarly to (19) we have

$$
\begin{aligned}
& \sum_{i=1}^{m} \mathbb{P}\left\{\sup _{t \in\left[c_{i}-\epsilon, d_{i}+\epsilon\right]} \frac{\chi_{\boldsymbol{b}}^{2}(t)}{w^{2}(t)}>u\right\}+\mathbb{P}\left\{\sup _{t \in \mathcal{T}_{\varepsilon}} \frac{\chi_{\boldsymbol{b}}^{2}(t)}{w^{2}(t)}>u\right\} \\
& \geq \mathbb{P}\left\{\sup _{t \in \mathcal{T}} \frac{\chi_{\boldsymbol{b}}^{2}(t)}{w^{2}(t)}>u\right\} \\
& \geq \sum_{i=1}^{m} \mathbb{P}\left\{\sup _{t \in\left[c_{i}, d_{i}\right]} \frac{\chi_{\boldsymbol{b}}^{2}(t)}{w^{2}(t)}>u\right\}-\sum_{1 \leq i<j \leq m} \mathbb{P}\left\{\sup _{t \in\left[c_{i}, d_{i}\right]} \frac{\chi_{\boldsymbol{b}}^{2}(t)}{w^{2}(t)}>u, \sup _{t \in\left[c_{j}, d_{j}\right]} \frac{\chi_{\boldsymbol{b}}^{2}(t)}{w^{2}(t)}>u\right\} .
\end{aligned}
$$


Next, we have from $\mathbf{F} 2$ that for $1 \leq i \leq m$

$$
\begin{aligned}
& \mathbb{P}\left\{\sup _{t \in\left[c_{i}, d_{i}\right]} \frac{\chi_{\boldsymbol{b}}^{2}(t)}{w^{2}(t)}>u\right\}=\mathbb{P}\left\{\sup _{t \in\left[c_{i}, d_{i}\right]} \chi_{\boldsymbol{b}}^{2}(t)>w^{2}\left(c_{1}\right) u\right\}, \\
& \mathbb{P}\left\{\sup _{t \in\left[c_{i}-\varepsilon, d_{i}+\varepsilon\right]} \frac{\chi_{\boldsymbol{b}}^{2}(t)}{w^{2}(t)}>u\right\} \leq \mathbb{P}\left\{\sup _{t \in\left[c_{i}-\varepsilon, d_{i}+\varepsilon\right]} \chi_{\boldsymbol{b}}^{2}(t)>w^{2}\left(c_{1}\right) u\right\} .
\end{aligned}
$$

It is noted that the result in Theorem 2.1 of [10] also holds when $g(t)=0$. Thus, it follows from that result, as $u \rightarrow \infty$,

$$
\begin{aligned}
\mathbb{P}\left\{\sup _{t \in\left[c_{i}, d_{i}\right]} \chi_{\boldsymbol{b}}^{2}(t)>w^{2}\left(c_{1}\right) u\right\} & \sim \prod_{j=k+1}^{n}\left(1-b_{j}^{2}\right)^{-1 / 2} \mathcal{H}_{\alpha} \int_{c_{i}}^{d_{i}}(C(t))^{1 / \alpha} d t\left(q\left(w^{2}\left(c_{1}\right) u\right)\right)^{-1} \Upsilon_{k}\left(w^{2}\left(c_{1}\right) u\right), \\
\mathbb{P}\left\{\sup _{t \in\left[c_{i}-\varepsilon, d_{i}+\varepsilon\right]} \chi_{\boldsymbol{b}}^{2}(t)>w^{2}\left(c_{1}\right) u\right\} & \sim \prod_{j=k+1}^{n}\left(1-b_{j}^{2}\right)^{-1 / 2} \mathcal{H}_{\alpha} \int_{c_{i}-\varepsilon}^{d_{i}+\varepsilon}(C(t))^{1 / \alpha} d t\left(q\left(w^{2}\left(c_{1}\right) u\right)\right)^{-1} \Upsilon_{k}\left(w^{2}\left(c_{1}\right) u\right) .
\end{aligned}
$$

Moreover, since

$$
\begin{aligned}
\mathbb{P} & \left\{\sup _{t \in\left[c_{i}, d_{i}\right]} \frac{\chi_{\boldsymbol{b}}^{2}(t)}{w^{2}(t)}>u, \sup _{t \in\left[c_{j}, d_{j}\right]} \frac{\chi_{\boldsymbol{b}}^{2}(t)}{w^{2}(t)}>u\right\} \\
& =\mathbb{P}\left\{\sup _{t \in\left[c_{i}, d_{i}\right]} \chi_{\boldsymbol{b}}^{2}(t)>w^{2}\left(c_{1}\right) u, \sup _{t \in\left[c_{j}, d_{j}\right]} \chi_{\boldsymbol{b}}^{2}(t)>w^{2}\left(c_{1}\right) u\right\},
\end{aligned}
$$

we have from Lemma 4.2 that, for all $u$ large,

$$
\mathbb{P}\left\{\sup _{t \in\left[c_{i}, d_{i}\right]} \frac{\chi_{\boldsymbol{b}}^{2}(t)}{w^{2}(t)}>u, \sup _{t \in\left[c_{j}, d_{j}\right]} \frac{\chi_{\boldsymbol{b}}^{2}(t)}{w^{2}(t)}>u\right\} \leq \exp \left(-\frac{\left(2 w\left(c_{1}\right) \sqrt{u}-Q_{i, j}\right)^{2}}{2(2+2 \eta)}\right), \quad 1 \leq i<j \leq m,
$$

with $Q_{i, j}$ 's some positive constants and $\eta \in(0,1)$. This implies that

$$
\sum_{1 \leq i<j \leq m} \mathbb{P}\left\{\sup _{t \in\left[c_{i}, d_{i}\right]} \frac{\chi_{\boldsymbol{b}}^{2}(t)}{w^{2}(t)}>u, \sup _{t \in\left[c_{j}, d_{j}\right]} \frac{\chi_{\boldsymbol{b}}^{2}(t)}{w^{2}(t)}>u\right\}=o\left(\left(q\left(w^{2}\left(c_{1}\right) u\right)\right)^{-1} \Upsilon_{k}\left(w^{2}\left(c_{1}\right) u\right)\right), \quad u \rightarrow \infty .
$$

Moreover, Lemma 4.1 gives that

$$
\mathbb{P}\left\{\sup _{t \in \mathcal{T}_{\varepsilon}} \frac{\chi_{\boldsymbol{b}}^{2}(t)}{w^{2}(t)}>u\right\}=o\left(\left(q\left(w^{2}\left(c_{1}\right) u\right)\right)^{-1} \Upsilon_{k}\left(w^{2}\left(c_{1}\right) u\right)\right), \quad u \rightarrow \infty
$$

Consequently, by letting $\varepsilon \rightarrow 0$ we conclude that the claim in (ii) is established. This completes the proof.

Acknowledgement: P. Liu was partially supported by the Swiss National Science Foundation Grant 200021166274 .

\section{REFERENCES}

[1] J. Albin and D. Jarušková, "On a test statistic for linear trend," Extremes, vol. 6, no. 3, pp. 247-258 (2004), 2003.

[2] D. Jarušková and V. Piterbarg, "Log-likelihood ratio test for detecting transient change," Statist. Probab. Lett., vol. 81, no. 5, pp. 552-559, 2011.

[3] G. Lindgren, "Slepian models for $\chi^{2}$-processes with dependent components with application to envelope upcrossings," J. Appl. Probab., vol. 26, no. 1, pp. 36-49, 1989.

[4] P. Liu and L. Ji, "Extremes of chi-square processes with trend," Probab. Math. Statist., vol. 36, no. 1, pp. 1-20, 2016.

[5] D. Konstantinides, V. Piterbarg, and S. Stamatovic, "Gnedenko-type limit theorems for cyclostationary $\chi^{2}$-processes," Lithuanian Mathematical Journal, vol. 44, no. 2, pp. 157-167, 2004.

[6] R. Adler and J. Taylor, Random fields and geometry. Springer Monographs in Mathematics, New York: Springer, 2007. 
[7] V. Piterbarg, Asymptotic methods in the theory of Gaussian processes and fields, vol. 148 of Translations of Mathematical Monographs. Providence, RI: American Mathematical Society, 1996. Translated from the Russian by V.V. Piterbarg, revised by the author.

[8] D. Cheng and Y. Xiao, "Excursion probability of Gaussian random fields on sphere," Bermoulli, vol. 22, pp. 1113-1130, 2016.

[9] D. Cheng and Y. Xiao, "The mean Euler characteristic and excursion probability of Gaussian random fields with stationary increments," Ann. Appl. A Probab., vol. 26, pp. 722-759, 2016.

[10] P. Liu and L. Ji, "Extremes of locally stationary chi-square processes with trend," Stochastic Process. Appl., vol. 127, no. 2, pp. 497-525, 2017.

[11] M. Csörgö, S. Csörgö, L. Horváth, and D. M. Mason, "Weighted empirical and quantile processes," Annals of Probability, vol. 14, no. 1, pp. 31-85, 1986.

[12] A. DasGupta, Asymptotic Theory of Statistics and Probability. Springer Texts in Statistics, Springer-Verlag New York, 2008.

[13] V. Piterbarg and V. Taran, "Exact asymptotics for large deviation probabilities of normalized Brownian bridge with applications to empirical processes," Computers Math. Applic., vol. 22, no. 7, pp. 85-91, 1991.

[14] L. Dümbgen, P. Kolesnyk, and R. A. Wilke, "Bi-log-concave distribution functions," Journal of Statistical Planning and Inference, vol. 184, pp. 1-17, 2017.

[15] R. Chicheportiche and J.-P. Bouchaud, "Weighted Kolmogorov-Smirnov test: Accounting for the tails," Phys. Rev. E, vol. 86, pp. 041-115, 2012.

[16] L. Bai, K. Dȩbicki, E. Hashorva, and L. Luo, "On generalised Piterbarg constants," Methodol Comput Appl Probab, vol. 20, pp. 137-164, 2018.

[17] L. Ji, P. Liu, and S. Robert, "Tail asymptotic behavior of the supremum of a class of chi-squre processes: with supplements," https://arxiv.org/pdf/1801.02486v2.pdf, 2019.

[18] N. Bingham, C. Goldie, and J. Teugels, Regular variation, vol. 27 of Encyclopedia of Mathematics and its Applications. Cambridge University Press, Cambridge, 1989.

[19] Z. Michina, "Remarks on Pickands theorem," Probability and Mathematical Statistics, vol. 37, no. 2, pp. 373-393, 2017.

Lanpeng Ji, School of Mathematics, University of Leeds, Woodhouse Lane, Leeds LS2 9JT, United Kingdom

E-mail address: 1.ji@leeds.ac.uk

Peng Liu, Department of Statistics and Actuarial Science, University of Waterloo, Canada

E-mail address: peng.liu1@uwaterloo.ca

Stephan Robert, Institute for Information and Communication Technologies, School of Business and Engineering Vaud (HEIG-VD), University of Applied Sciences of Western Switzerland, Switzerland

E-mail address: Stephan.Robert@heig-vd.ch 